\title{
Technical note: High-throughput method for antifungal activity screening in a cheese-mimicking model
}

\author{
Lucille Garnier, ${ }^{\star} \dagger$ Marcia Leyva Salas, ${ }^{\star} \dagger$ Nicolas Pinon,† Norman Wiernasz, ${ }^{*}$ Audrey Pawtowski, ${ }^{*}$ \\ Emmanuel Coton, ${ }^{*}$ Jérôme Mounier, ${ }^{*}$ and Florence Valence ${ }^{1}$ \\ *Université de Brest, EA 3882, Laboratoire Universitaire de Biodiversité et Ecologie Microbienne, ESIAB, Technopôle Brest-Iroise, \\ 29280 Plouzané, France \\ †STLO, Agrocampus Ouest, INRA, 35000 Rennes, France
}

\section{ABSTRACT}

In this study, we developed a high-throughput antifungal activity screening method using a cheese-mimicking matrix distributed in 24-well plates. This method allowed rapid screening of a large variety of antifungal agent candidates: bacterial fermented ingredients, bacterial isolates, and preservatives. Using the proposed method, we characterized the antifungal activity of 44 lactic acid bacteria (LAB) fermented milk-based ingredients and $23 \mathrm{LAB}$ isolates used as protective cultures against 4 fungal targets (Mucor racemosus, Penicillium commune, Galactomyces geotrichum, and Yarrowia lipolytica). We also used this method to determine the minimum inhibitory concentration of a preservative, natamycin, against 9 fungal targets. The results underlined the strain-dependency of LAB antifungal activity, the strong effect of fermentation substrate on this activity, and the effect of the screening medium on natamycin minimum inhibitory concentration. Our method could achieved a screening rate of 1,600 assays per week and can be implemented to evaluate antifungal activity of microorganisms, fermentation products, or purified compounds compatible with dairy technology.

Key words: high-throughput screening, antifungal activity, cheese model

\section{Technical Note}

Fungal spoilage of dairy products remains a major concern for dairy manufacturers despite the use of preventive and control approaches, including the use of chemical preservatives. Among methods that are increasingly used by industries for microbial contaminant control, biopreservation is gaining increasing attention due to strong societal (Gerez et al., 2013) and legisla-

Received July 18, 2017.

Accepted November 30, 2017.

${ }^{1}$ Corresponding author: florence.valence-bertel@inra.fr tive (Fuselli et al., 2012; Stratford et al., 2013) demand for preservative-free products. Biopreservation can be defined as using natural or added microbiota or their antimicrobial compounds to preserve a food product (in terms of safety and quality) and possibly extend its shelf life (Stiles, 1996). Lactic acid bacteria (LAB) are well known for their ability to exhibit antifungal activity (Schillinger and Villareal, 2010; Wulijideligen and Taku, 2011; Cheong et al., 2014) and protective cultures containing LAB, such as FreshQ (consisting of Lactobacillus paracasei and Lactobacillus rhamnosus strains; Chr. Hansen, Hørsholm, Denmark) or the HOLDBAC series (consisting of various lactobacilli and propionibacteria; Danisco/DuPont, Madison, WI), are currently used in dairy products for their antifungal properties (Varsha and Nampoothiri, 2016). Recently, Inglin et al. (2015) developed a high-throughput screening method to detect antimicrobial (including antifungal) activities using an agar-spot assay in 24-well plates. This method enabled screening of 2,000 assays per day. However, despite being a useful primary high-throughput screening method for developing protective cultures, this antifungal screening assay used de Man, Rogosa, and Sharpe (MRS) agar medium, which can strongly affect expression of antifungal properties and antifungal molecule activity. Indeed, as mentioned in several articles (Stiles et al., 2002; Delavenne et al., 2012; Le Lay et al., 2016), MRS contains acetate, which may reinforce LAB antifungal activity and artificially inflate the number of active isolates. However, assays applied to real food matrices (e.g., dairy product models such as yogurt) can rapidly become labor intensive, thereby reducing the number of isolates that can be screened to detect strains with antifungal activity (Lynch et al., 2014; Delavenne et al., 2015). In addition, these methods do not allow large-scale and rapid screening of antifungal cultures or compounds.

In the present study, we present a high-throughput screening assay for antifungal activity in a cheesemimicking matrix distributed in a 24 -well plate. The 
proposed method allowed testing the antifungal activity of a large variety of microbial protective compounds: bacterial fermentation products, bacterial cultures used as adjunct cultures, and purified antifungal preservatives. To prepare the cheese-mimicking matrix, we used a standardized UF milk retentate $(3.4 \times ; 244 \mathrm{~g} / \mathrm{kg}$ of DM, $65.8 \mathrm{~g} / \mathrm{kg}$ total fat, $116.2 \mathrm{~g} / \mathrm{kg}$ of total $\mathrm{N}, \mathrm{pH} 6.53)$ prepared according to Hannon et al. (2006). Briefly, whole raw cow milk was heated to $50^{\circ} \mathrm{C}$ and skimmed using a cream separator (Westfalia, Handelsweg, the Netherlands). Skim milk was then microfiltered $(0.8-\mu \mathrm{m}$ Sterilox GP membrane, Pall Corp., Port Washington, $\mathrm{NY}$ ) at $50^{\circ} \mathrm{C}$ using the pilot equipment GP7 (Brenet, Mamirolle, France) and cream was heat-treated for $2 \mathrm{~min}$ at $95^{\circ} \mathrm{C}$ followed by fat standardization $(20 \mathrm{~g} /$ $\mathrm{kg}$ final concentration). The retentate was ultrafiltered at $0.02 \mu \mathrm{m}$ at $50^{\circ} \mathrm{C}$ (T.I.A., Bollene, France) and sterilized sodium chloride (Sogebul, Sainte-Maure de Touraine, France) was added to reach $0.7 \%$ (wt/ wt). The salted retentate was heat-treated for $2 \mathrm{~min}$ at $95^{\circ} \mathrm{C}$ (Microthermics, Raleigh, NC), distributed in 1-L sterile bottles, and kept at $-20^{\circ} \mathrm{C}$ until use. Before plate preparation, $10 \mathrm{~mL} / \mathrm{L}$ of a $\mathrm{pH}$ indicator (sterile solution of litmus $50 \mathrm{~g} / \mathrm{L}), 10^{6} \mathrm{cfu} / \mathrm{L}$ of commercial starter MA016 (Lactococcus lactis ssp. cremoris and $L$. lactis ssp. lactis, Elimeca, Thoissey, France), and 1.5 $\mathrm{mL} / \mathrm{L}$ of $5 \times$ diluted and filtered $(0.22 \mu \mathrm{m})$ rennet (Danisco, Dangé Saint-Romain, France) were added to the thawed retentate. After vigorous homogenization for 1 min, the retentate was distributed into 24 -well plates $(2$ $\mathrm{mL} /$ well) and incubated for $1 \mathrm{~h}$ at $30^{\circ} \mathrm{C}$ and then for 3 $\mathrm{d}$ at $20^{\circ} \mathrm{C}$, leading to the formation of a "mini-cheese" ( $\mathrm{pH} 5)$ in each well. The exudate was then removed from the surface of each mini-cheese and plates were stored at $12^{\circ} \mathrm{C}$ until use.

Using this cheese-mimicking matrix, we screened the antifungal activity of $44 \mathrm{LAB}$ fermented milk-based products and $23 \mathrm{LAB}$ isolates used as protective cultures against 4 fungal targets. The LAB were obtained from the culture collections of CIRM-BIA (Centre International de Ressources Microbienne-Bactéries d'Intérêt Alimentaire, Rennes, France) and LUBEM (Laboratoire Universitaire de Biodiversité et Ecologie Microbienne, Plouzané, France). For fermentation product preparation, a 10\%-reconstituted low-heat milk supplemented with $45 \%$ anhydrous milk fat and $0.5 \%$ litmus (LH medium) was sterilized for $30 \mathrm{~min}$ at $110^{\circ} \mathrm{C}$. Then, it was individually inoculated with a suspension of the LAB to be tested $(1 \% \mathrm{vol} / \mathrm{vol})$, obtained after 2 subcultures of $24 \mathrm{~h}$ at $30^{\circ} \mathrm{C}$ in MRS broth (Difco, Le Pont de Claix, France). The LH medium inoculated with $\mathrm{LAB}$ was then incubated for $20 \mathrm{~h}$ at $30^{\circ} \mathrm{C} ; 100 \mu \mathrm{L}$ of the resulting fermentation product was then deposited on the surface of each mini-cheese. After drying for $2 \mathrm{~h}$ at room temperature under laminar air flow, plates were surface inoculated with 1 of 4 fungal targets: Mucor racemosus UBOCC-A-116002, Penicillium commune UBOCC-A-116003, Galactomyces geotrichum UBOCC-A-216001, or Yarrowia lipolytica UBOCCA-216006, which were previously isolated from dairy products (Garnier et al., 2017) and obtained from the Université de Bretagne Occidentale Culture Collection (UBOCC, Plouzané, France). Except for yeasts that were first cultured in potato dextrose broth, $10 \mu \mathrm{L}$ of suspension containing $5 \times 10^{3}$ spores or cells $/ \mathrm{mL}$, obtained as previously described (Delavenne et al., 2012), was spotted at the center of each mini-cheese ( 1 tested fungus/plate). Plates were then incubated at $12^{\circ} \mathrm{C}$ for 5,6 , and $8 \mathrm{~d}$ for $M$. racemosus, G. geotrichum, and $P$. commune and $Y$. lipolytica, respectively. Antifungal activity was then determined by visually evaluating fungal growth compared with a negative control without any fermentation product (Figure 1). To test the antifungal activity of LAB isolates for potential use as protective cultures, we applied the same methodology except that LAB isolates to be tested were individually suspended in sterilized milk to reach a final concentration of $10^{7} \mathrm{cfu} / \mathrm{mL}$ of retentate and inoculated concomitantly with the commercial starter MA016, $\mathrm{pH}$ indicator, and rennet before distribution in 24-well plates. In this context, $23 \mathrm{LAB}$ cultures were tested in duplicate against the same fungal targets as those described above, after 2 pre-cultures for $24 \mathrm{~h}$ at $30^{\circ} \mathrm{C}$ in MRS broth. Incubation to obtain the mini-cheeses was performed as described above. Plates were then surface-inoculated with 1 of the 4 fungal targets and antifungal activity was evaluated as described above.

All tested fungal species grew well in the cheesemimicking model (Supplemental Figure S1; https://doi .org/10.3168/jds.2017-13518) and the results obtained for both replicates were similar, confirming the suitability of this matrix to sustain fungal growth and the reproducibility of the screening method. Among the 46 tested LAB fermentation products, 25 showed antifungal activity against at least one fungal target, and 22 out of the 23 tested protective cultures showed antifungal activity against at least one target. More precisely, intermediate antifungal activities against at least one fungal target were observed for $14(30 \%)$ fermentation products and $12(55 \%)$ isolates, whereas complete inhibition against at least one fungal target was only observed for LAB isolates $(\mathrm{n}=10 ; 45 \%$; Table $1)$. The most active $(++$ and +++$)$ isolates or fermentation products corresponded to Lactobacillus casei, Lactobacillus plantarum, Lactobacillus brevis, Lactobacillus paracasei, Leuconostoc mesenteroides, and, to a lesser extent, Lb. rhamnosus. Antifungal activity varied depending on the utilization mode (protective culture or 


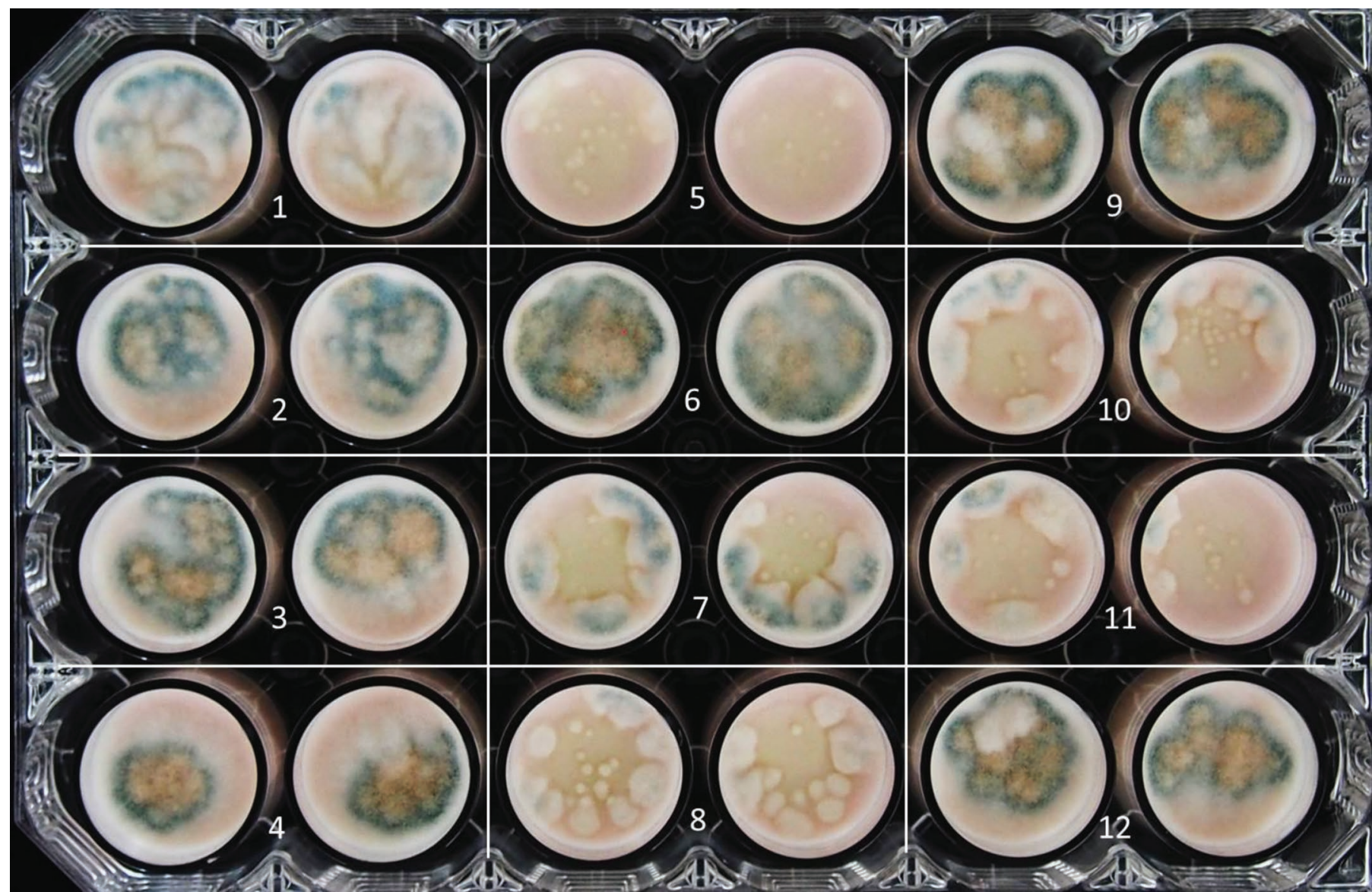

Figure 1. Visual evaluation of fungal growth to determine antifungal activity. Photograph of a 24-well plate showing the antifungal activity of 11 fermentation products from lactobacilli strains (tested in duplicate) against Penicillium commune UBOCC-A-116003 (50 spores/well) in mini-cheeses incubated for $8 \mathrm{~d}$ at $12^{\circ} \mathrm{C}$. Lactobacilli strains: Lactobacillus plantarum L89 (well 1) and L96 (8); Lactobacillus buchneri L90 (2); Lactobacillus reuteri L91 (3), L92 (4), L94 (6), and L97 (9); Lactobacillus casei L95 (7); Lactobacillus paracasei L93 (5); and Lactobacillus rhamnosus CIRM-BIA 607 (10) and CIRM-BIA 909 (11) fermentation products; negative control (without fermentation product) (12). No antifungal activity (-) observation in positions $2,3,4,6,9$; weak activity $(+)$ in position 1 ; intermediate antifungal activities $(++)$ in positions $5,7,8,10$ and 11. When complete inhibition was observed, a " $+++"$ score was attributed to the corresponding isolate.

fermentation product). For example, for Lb. plantarum strains tested both for their fermentation products and as protective culture, fermentation products showed no activity against $M$. racemosus but could inhibit the latter when used as protective cultures (Table 1). In contrast, $L b$. brevis CIRM-BIA 608 showed activity only against $M$. racemosus and $P$. commune when used as a fermentation product. None of the tested isolates of Lactobacillus pentosus, Lactobacillus sakei, or Leuconostoc citreum showed any activity (Table 1). As expected, within a species, not all tested isolates showed the same antifungal activity. For example, each tested $L b$. casei isolate showed antifungal activity but the inhibited fungal target and the obtained antifungal scores were different among them, confirming that antifungal activity is a strain-dependent trait, as suggested by Cortés-Zavaleta et al. (2014). Finally, under the tested conditions, $P$. commune was the most frequently inhibited target, followed by $M$. racemosus and G. geotrichum. None of the strains used to produce fermentation products was able to inhibit growth of $Y$. lipolytica, whereas only 3 strains used as protective cultures (Lb. brevis CIRM-BIA 128, Lb. plantarum L244, and Leu. mesenteroides CIRM-BIA 1187) showed intermediate antifungal activity against this target (Table 1). Overall, antifungal activity of the tested strains was higher when they were used as protective cultures; indeed, all isolates did show antifungal activity. The reproducibility of antifungal activities obtained with 2 batches of mini-cheeses for 2 protective cultures and fermentates was evaluated (Supplemental Figure S2; https://doi.org/10.3168/jds.2017-13518). The obtained antifungal activities showed a good reproducibility despite slight variation.

The proposed method was also used to evaluate resistance to natamycin (a natural antibiotic produced by 
Streptomyces natalensis used for fungal control on cheese surface) of 9 fungi, including those previously mentioned as well as Phoma pinodella UBOCC-A-116004, Candida parapsilosis UBOCC-A-216002, Meyerozyma guilliermondii UBOCC-A-216003, Trichosporon asahii UBOCC-A-216005, and Rhodotorula mucilaginosa UBOCC-A-216004 (Garnier et al., 2017). Natamycin resistance was tested in a similar concentration range (from 0 to $0.2 \mathrm{mg} / \mathrm{dm}^{2}$ ) as that used by Garnier et al. (2017). Natamycin was deposited on the mini-cheese surface, in duplicate, using $100 \mu \mathrm{L}$ of a stock solution followed by fungal inoculation on the cheese surface as described above. The MIC was determined as the concentration for which no visible growth occurred

Table 1. Lactic acid bacteria used as dairy ingredient (fermentation product) or protective culture for the antifungal assay

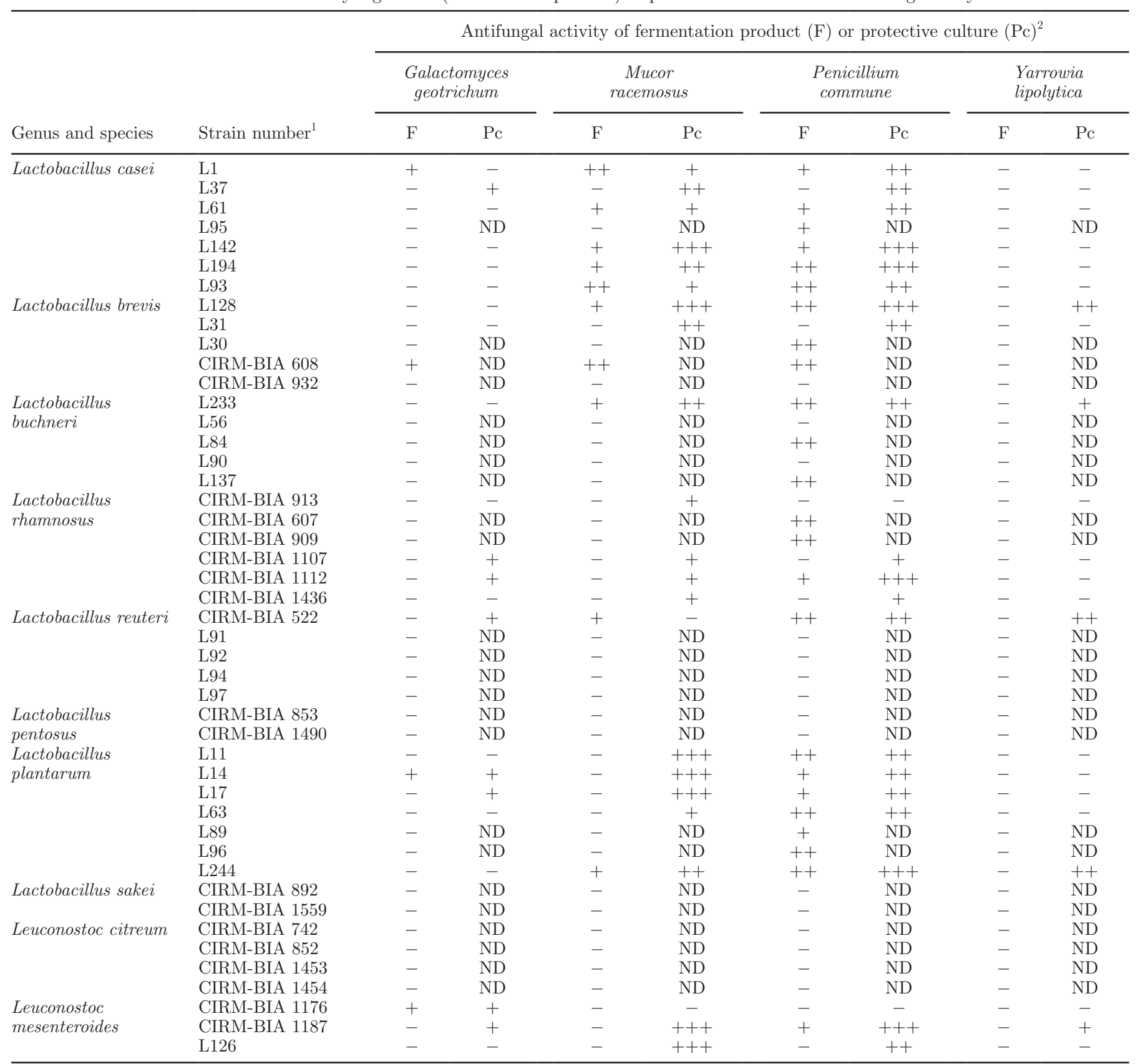

${ }^{1} \mathrm{~L}=$ isolates from Laboratoire Universitaire de Biodiversité et Ecologie Microbienne (Plouzané, France); CIRM-BIA = isolates from Centre International de Ressources Microbienne-Bactéries d'Intérêt Alimentair (Rennes, France).

${ }^{2}$ Where $-=$ no antifungal activity $;+=$ weak antifungal activity $;++=$ intermediate antifungal activity $;+++=$ complete inhibition; ND $=$ not determined (not tested as protective culture). 
Table 2. Minimal inhibitory concentrations (g/L) of natamycin in model cheese versus potato dextrose agar (Garnier et al., 2017) for 9 isolates of representative spoilage fungal species encountered in dairy products

\begin{tabular}{|c|c|c|c|c|}
\hline \multirow[b]{2}{*}{ Fungal species } & \multirow[b]{2}{*}{ Strain number ${ }^{1}$} & \multirow[b]{2}{*}{ Isolation source } & \multicolumn{2}{|c|}{$\mathrm{MIC}^{2}$} \\
\hline & & & Model cheese & Potato dextrose agar \\
\hline Mucor racemosus & UBOCC-A-116002 & Semi-hard cheese & $0.04(0.2)$ & $0.1(0.5)$ \\
\hline Penicillium commune & UBOCC-A-116003 & Semi-hard cheese & $0.06(0.3)$ & $0.06(0.3)$ \\
\hline Phoma pinodella & UBOCC-A-116004 & Cream cheese & $0.08(0.4)$ & $0.1(0.5)$ \\
\hline Candida parapsilosis & UBOCC-A-216002 & Yogurt & $0.08(0.4)$ & $0.1(0.5)$ \\
\hline Trichosporon asahii & UBOCC-A-216005 & Fresh cheese & $0.04(0.2)$ & $0.1(0.5)$ \\
\hline Yarrowia lipolytica & UBOCC-A-216006 & Semi-hard cheese & $0.16(0.8)$ & $>0.2(>1.0)^{3}$ \\
\hline
\end{tabular}

${ }^{1}$ Université de Bretagne Occidentale Culture Collection (UBOCC, Plouzané, France).

${ }^{2}$ With $\mathrm{mg} / \mathrm{dm}^{2}$ in parentheses.

${ }^{3}$ Highest tested concentration.

after the incubation period (Table 2). The obtained MIC values were then compared with those obtained by Garnier et al. (2017) in potato dextrose agar at $\mathrm{pH}$ 5 . Independently of the fungal target, natamycin MIC were generally slightly lower in the cheese model than in potato dextrose agar. This result may be explained by differences in the physiological status of the fungal targets as well as by the fact that other compounds present in the cheese may enhance natamycin activity. For example, lactic acid produced by lactococci starter cultures could act synergistically with natamycin.

Mucor racemosus and T. asahii were the most sensitive fungi to natamycin with a MIC of $0.04 \mathrm{mg} / \mathrm{dm}^{2}$, followed by $P$. commune $\left(0.06 \mathrm{mg} / \mathrm{dm}^{2}\right.$; Table 2). Yarrowia lipolytica showed the highest resistance to natamycin with a MIC of $0.16 \mathrm{mg} / \mathrm{dm}^{2}$, which is very close to the maximum authorized concentration $(0.2 \mathrm{mg} /$ $\mathrm{dm}^{2}$ ) that can be used in dairy products within the European Union.

The main objective of this work was to develop a high-throughput screening method to evaluate antifungal activities of various antifungal agent candidates (bioprotective cultures, fermentation products, or purified molecules) in a matrix as close as possible to real dairy products, such as cheese, in terms of physical, chemical, and biological composition. The proposed method, based on the use of a cheese mimicking-model distributed in 24-well plates, could achieve a screening rate of 1,600 assays per week, is easy to implement, and does not require complex and high-cost equipment. Such a screening matrix, closer to an actual dairy product, makes antifungal activity screening more robust in regards to scale-up validation, which follows the screening step. Even though the gross composition of the retentate used to make mini-cheeses was verified in 3 batches $(252 \pm 8 \mathrm{~g} / \mathrm{kg} \mathrm{DM}, 67.8 \pm 1.9 \mathrm{~g} / \mathrm{kg}$ fat, $113.9 \pm 2.08 \mathrm{~g} / \mathrm{kg} \mathrm{N}$ content, $\mathrm{pH} 6.53 \pm 0.02)$, slight variations in observed antifungal activities could occur from one retentate batch to another. Indeed, minor compound (e.g., micronutrient) variability is expected due to seasonal variation in milk composition but such variability was not verified in the context of this study. In addition, by determining the number of pixels represented by the targeted fungi on the cheese surface, estimation of fungal growth could be automated using image analysis, yielding a quantitative estimation of fungal growth. In conclusion, the developed method offers new perspectives for developing new biopreservation solutions.

\section{ACKNOWLEDGMENTS}

This work was financially supported by the Conseils Régionaux de Bretagne (grant no. 13008651) and Pays de la Loire (grant no. 2014-01081) in the framework of the PROFIL interregional project under the scientific coordination of INRA (J. Leonil) and managed by the Bretagne Biotechnologies Alimentaires industrial association (Rennes, France).

\section{REFERENCES}

Cheong, E. Y. L., A. Sandhu, J. Jayabalan, T. T. Kieu Le, and N. T. Nhiep., H. TMy Ho, J., N. Zwielehner, Bansal, and M. S. Turner. 2014. Isolation of lactic acid bacteria with antifungal activity against the common cheese spoilage mould Penicillium commune and their potential as biopreservatives in cheese. Food Control 46:91-97.

Cortés-Zavaleta, O., A. López-Malo, A. Hernández-Mendoza, and H. S. García. 2014. Antifungal activity of lactobacilli and its relationship with 3-phenyllactic acid production. Int. J. Food Microbiol. 173:30-35.

Delavenne, E., S. Cliquet, C. Trunet, G. Barbier, J. Mounier, and G. Le Blay. 2015. Characterization of the antifungal activity of Lactobacillus harbinensis K.V9.3.1Np and Lactobacillus rhamnosus K.C8.3.1I in yogurt. Food Microbiol. 45:10-17.

Delavenne, E., J. Mounier, F. Deniel, G. Barbier, and G. Le Blay. 2012. Biodiversity of antifungal lactic acid bacteria isolated from 
raw milk samples from cow, ewe, and goat over one-year period. Int. J. Food Microbiol. 155:185-190.

Fuselli, F., C. Guarino, A. La Mantia, L. Longo, A. Faberi, and R. M. Marianella. 2012. Multi-detection of preservatives in cheeses by liquid chromatography-tandem mass spectrometry. J. Chromatogr. B Analyt. Technol. Biomed. Life Sci. 906:9-18.

Garnier, L., F. Valence, A. Pawtowski, L. Auhustsinava-Galerne, N. Frotté, F. Deniel, E. Coton, and J. Mounier. 2017. Diversity of spoilage fungi associated with various French dairy products. Int. J. Food Microbiol. 241:191-197.

Gerez, C. L., M. J. Torres, G. Font de Valdez, and R. Rollan. 2013 Control of spoilage fungi by lactic acid bacteria. Biol. Control 64:231-237.

Hannon, J. A., S.-M. Deutsch, M.-N. Madec, J.-Y. Gassi, M.-P. Chapot-Chartier, and S. Lortal. 2006. Lysis of starters in UF cheeses: Behaviour of mesophilic lactococci and thermophilic lactobacilli. Int. Dairy J. 16:324-334.

Inglin, R. C., M. J. Stevens, L. Meile, C. Lacroix, and L. Meile. 2015. High-throughput screening assays for antibacterial and antifungal activities of Lactobacillus species. J. Microbiol. Methods 114:26-29.

Le Lay, C., J. Mounier, V. Vasseur, A. Weill, G. Le Blay, G. Barbier, and E. Coton. 2016. In vitro and in situ screening of lactic acid bacteria and propionibacteria antifungal activities against bakery product spoilage molds. Food Control 60:247-255.
Lynch, K. M., A. M. Pawlowska, B. Brosnan, A. Coffey, E. Zannini, A. Furey, P. L. H. McSweeney, D. M. Waters, and E. K. Arendt. 2014 Application of Lactobacillus amylovorus as an antifungal adjunct to extend the shelf-life of Cheddar cheese. Int. Dairy J. 34:167-173.

Schillinger, U., and J. V. Villarreal. 2010. Inhibition of Penicillium nordicum in MRS medium by lactic acid bacteria isolated from foods. Food Control 21:107-111.

Stiles, J., S. Penkar, M. Plocková, J. Chumchalová, and L. B. Bullerman. 2002. Antifungal activity of sodium acetate and Lactobacillus rhamnosus. J. Food Prot. 65:1188-1191.

Stiles, M. E. 1996. Biopreservation by lactic acid bacteria. Antonie van Leeuwenhoek 70:331-345.

Stratford, M., H. Steels, G. Nebe-von-Caron, M. Novodvorska, K. Hayer, and D. B. Archer. 2013. Extreme resistance to weak-acid preservatives in the spoilage yeast Zygosaccharomyces bailii. Int. J. Food Microbiol. 166:126-134.

Varsha, K. K., and K. M. Nampoothiri. 2016. Appraisal of lactic acid bacteria as protective cultures. Food Control 69:61-64.

Wulijideligen, S., and M. Taku. 2011. Screening and identification of lactic acid bacteria from Airag for antifungal activity. J. Anim. Vet. Adv. 10:2751-2757. 